\title{
KLAUSELRHYTHMUS IN DEN DIREKTEN REDEN DES CORPUS CAESARIANUM
}

Summary: Comparing the prose-rhythm in the direct speeches of the Corpus Caesarianum produces another proof for Caesar being not the author of the writings Bellum Alexandrinum, Bellum Africum and Bellum Hispaniense.

Key words: prose-rhythm, clausulae, Caesar, Pseudo-Caesar, direct speeches

\section{EINLEITUNG}

Die Sprache hat in Gesellschaften einen besonderen Stellenwert inne. Das vergegenwärtigt uns z. B. die Politik von Tag zu Tag auf ein Neues. Nicht umsonst trägt eine Ausgabe der Politischen Meinung den Titel Sprache und Politik. ${ }^{1}$

Das Verständlichste an der Sprache ist nicht das Wort selber, sondern Ton, Stärke, Modulation, Tempo, mit denen eine Reihe von Worten gesprochen wird - kurz die Musik hinter den Worten, die Leidenschaft hinter dieser Musik, die Person hinter dieser Leidenschaft: alles das also, was nicht geschrieben [Hervorhebung weggelassen; d. Verf.] werden kann. ${ }^{2}$

In diesem Sinne sprechen H. und H. Huchzermeyer in ihrem Aufsatz Die Bedeutung des Rhythmus in der Musiktherapie der Griechen von der Frühzeit bis zum Beginn des Hellenismus dem sprachlichen Rhythmus im antiken Griechenland eine magisch-

${ }^{1}$ Vgl. Vogel, B. (Hrsg.): Sprache und Politik. Wolfgang Bergsdorf zum 70. Geburtstag [Die Politische Meinung Nr. 504], 2011.

${ }^{2}$ NiETZSCHE, F.: Nachgelassene Werke. Unveröffentlichtes aus der Zeit der Fröhlichen Wissenschaft und des Zarathustra (1881-1886). Nietzsches Werke. Bd. XII (Bd. IV der zweiten Abteilung). Leipzig $1919^{3}, 334$. 
heilende Wirkung $\mathrm{zu},{ }^{3}$ während Stroh in seiner Monographie Die Macht der Rede. Eine kleine Geschichte der Rhetorik im alten Griechenland und Rom seine propagandistisch-manipulativen Effekte in der lateinischen Sprache betont. ${ }^{4}$

Wie die Sprache, d. h. Textaufbau, Wortwahl, Satzbau und eben auch der sprachliche Rhythmus, schon in der Antike maßgeblich für das politische Auftreten und Wirken war, so ist sie es noch heute. Natürlich haben die politischen Grundstrukturen und damit der Anspruch an eine politische Rede seitdem Änderungen erfahren. ${ }^{5}$ Dennoch: Die Sprache hat ihre Macht, speziell in der Politik, aber auch im Allgemeinen, bis heute nicht verloren. Nur durch sie können Anliegen zum Ausdruck gebracht und Erklärungen abgegeben werden. Nur durch sie kann ein Redner (oder allgemein ein Schriftsteller ${ }^{6}$ ) etwas propagieren und seine Zuhörer manipulieren.

Propaganda und Manipulation spielen im schriftstellerischen Wirken Caesars und seiner Fortsetzer eine große Rolle, wollten sie doch mit ihren Werken bestimmte militärische Operationen rechtfertigen.

[...] [D]iese Bücher sind nicht einfach Tatenberichte, sondern zugleich Praktizierung von Politik mit anderen - eben literarischen - Mitteln, Mitteln übrigens, die zu allen Zeiten und bis heute oft genug angewendet wurden und als völlig legitim zu betrachten sind, ${ }^{7}$

schreibt Richter in diesem Zusammenhang über die Kommentarien Caesars. Caesar war ein psychologisch versierter Schriftsteller. Er wusste genau, mit welchen Worten und mit welchem Stil er Rezipienten für seine Sache gewinnen konnte. ${ }^{8}$

Motivation für die folgenden Untersuchungen ist der Forschungsansatz, ob auch unter klauselrhythmischen Aspekten eine Antwort auf die in der Wissenschaft auf-

${ }^{3}$ Vgl. HuChZermeyer, H. - HuChZermeyer, H.: Die Bedeutung des Rhythmus in der Musiktherapie der Griechen von der Frühzeit bis zum Beginn des Hellenismus. ZWG 58 (1974) 113-148.

${ }^{4}$ Vgl. STROH, W.: Die Macht der Rede. Eine kleine Geschichte der Rhetorik im alten Griechenland und Rom. Berlin 2011, 380. Über den antiken Prosarhythmus schreiben neben anderen antiken

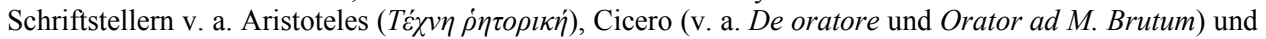
Quintilian (Institutio oratoria). Für eine ausführliche Darstellung des antiken Prosarhythmus vgl. LAUsBERG, H.: Handbuch der literarischen Rhetorik. Eine Grundlegung der Literaturwissenschaft. Stuttgart $2008^{4}, 479-507$ (§§ 977-1054).

${ }^{5}$ Vgl. STROH (Anm. 4) 515-517.

${ }^{6}$ Norden verwendet in diesem Sinne den Begriff , antike Kunstprosa“ und schreibt: „Die Rhetorik konnte bei der zentralen Stellung, die sie im antiken Denken und Handeln einnahm, nicht beschränkt bleiben auf die eigentlich gesprochene Rede, sondern mit Notwendigkeit erstreckte sie im Lauf der Zeit ihren Einflu[ss] auf alle Literaturgattungen, [...]" Vgl. NORDEN, E.: Die antike Kunstprosa. Vom VI. Jahrhundert v. Chr. bis in die Zeit der Renaissance. Bd. I. Stuttgart-Leipzig $1995^{10}$, Neudruck der 3. Auflage von 1915 , 9. Somit weisen nicht nur antike Reden rhythmische Strukturen auf, sondern auch antike prosaische Schriften anderer Genres - auch die Historiographie.

${ }^{7}$ RiCHTER, W.: Caesar als Darsteller seiner Taten. Eine Einführung [Bibliothek der klassischen Altertumswissenschaften, N. F., Reihe 2, Bd. LXI]. Heidelberg 1977, 39.

${ }^{8}$ Vgl. Collins, J. H.: Propaganda, Ethics, and Psychological Assumptions in Caesar's Writings. Diss. Frankfurt a. M. 1952; Collins, J. H.: Caesar und die Verführung der Macht. Historia 4 (1955) 445-465 (= RASMUSSEN, D. [Hrsg.]: Caesar [Wege der Forschung 67]. Darmstadt 1980, 379-412); KNOCHE, U.: Caesars Commentarii, ihr Gegenstand und ihre Absicht. Gymnasium 58 (1951) 139-160 (= Wege der Forschung 67, 224-254); STEVENS, C. E.: The Bellum Gallicum as a Work of Propaganda. REL 11 (1952) 3-18. 
geworfene Frage nach dem Verfasser bzw. den Verfassern des Corpus Caesarianum ${ }^{9}$ gegeben werden kann. Hierbei werden die direkten Reden im Corpus Caesarianum nach klauselrhythmischen Gesichtspunkten analysiert und verglichen.

Im Folgenden werden nach einer knappen Einführung in den bisherigen Stand der Forschung zunächst der Forschungsgegenstand sowie die eigene Methodik erläutert, bevor anschließend klauselrhythmisch analysierte Textbeispiele sowie die erreichten Ergebnisse präsentiert werden.

\section{FORSCHUNGSSTAND}

Der Prosarhythmus in den caesarischen Schriften wurde bisher, im Kontrast zu den zahlreichen Untersuchungen zu Caesars Kommentarien allgemein, nur recht wenig erforscht. Während Norden Caesar jegliche absichtliche Verwendung von Prosarhythmus in dessen Schriften abspricht, ${ }^{10}$ gesteht Ceci ihm diese zumindest in dessen direkten Reden zu. ${ }^{11}$ Er legt uns hierzu eine klauselrhythmische Analyse der Rede des Critognatus im Bellum Gallicum vor. ${ }^{12}$ Darauf aufbauend untersucht Holtz den Redestil Caesars. In diesem Zusammenhang liefert er uns klauselrhythmische Analysen zur Rede des Critognatus im Bellum Gallicum sowie zu der zweiten Rede des Curio im Bellum civile. ${ }^{13}$ De Groot greift u. a. Holtz' Untersuchungen auf, wenn er zum Prosarhythmus bei Caesar Folgendes schreibt:

Dafür, da[ss] er metrisch redete, ist noch überzeugender als die rednerischen und grammatischen Fragmente die Tatsache, da[ss] sogar seine [C]ommentarii [eigene Hervorhebung; d. Verf.], die doch die objektive Farbe unseres modernen Historienstils anstreben und in erster Linie darauf berechnet sind, durch das Fehlen jeder rhetorischen Kunstmittel zu überzeugen [Hervorhebung weggelassen; d. Verf.], nicht ganz unmetrisch sind, obgleich sich ihre metrischen Tendenzen nur statistisch, nicht etwa durch metrische Analysen nachweisen lassen. Deutlich metrisch aber sind (eine wichtige Entdeckung von Holtz) einige Reden, darunter diejenige des Critognatus. Ich mache darauf aufmerksam, da[ss] die Metrik dieser Rede a fortiori ganz dieselben Eigentümlichkeiten aufweist, wie die nicht rednerischen Partien des Bellum civile [eigene Hervorhebung; d. Verf.], also wohl auch des Bellum Gallicum [eigene Hervorhebung; d. Verf.]; diese individuellen Eigentümlichkeiten liegen, wie so oft, nicht so sehr in

\footnotetext{
${ }^{9}$ Die Bezeichnung, Corpus Caesarianum “ umfasst hier die Kommentarien Caesars und die pseudocaesarischen Schriften, anders als bei von Albrecht (vgl. vON ALBRECHT, M.: Geschichte der römischen Literatur. Von Andronicus bis Boethius und ihr Fortwirken. Bd. I. Berlin-Boston 201233 , 361-363).

${ }^{10} \mathrm{Vgl}$. NORDEN (Anm. 6) 939.

${ }^{11}$ Vgl. CeCI, L.: Il Ritmo delle Orazioni di Cicerone. Bd. I: La Prima Catilinaria. Turin u. a. 1905, 66.

${ }^{12}$ Vgl. CeCI (Anm. 11) 68-70.

${ }^{13}$ Vgl. HoltZ, L.: C. Iulius Caesar quo usus sit in orationibus dicendi genere. Diss. Jena 1913, $31-38$.
} 
der Vorliebe für gewisse Formen, sondern in den Frequenzverhältnissen: [B]esonders die Formen $-\mathrm{v}--[\mathrm{x}]$ und $-\mathrm{v}--\mathrm{v}-[\mathrm{x}]$ (beide häufiger als bei Cicero!), dazu die Form - $-[\mathrm{x}]$, die sonst in der metrischen Prosa gemieden ist (hier fast normal), treten stark hervor; dagegen tritt die Form $-\mathrm{v}-\ldots \mathrm{v}[\mathrm{x}]$ wie in der älteren Periode Ciceros etwas zurück; [...]. Diese Übereinstimmung zwischen den Reden und den sonstigen Partien ist bedeutsam für das antike Kompositionsgefühl, das nach unserer Auffassung stärker im Stil als im Inhalt die Einheitlichkeit sucht, dazu für die Tatsache, da[ss] die Metrik fast ganz zu etwas Mechanischem geworden ist, und besonders dafür, da[ss] jetzt in Rom wie in der griechischen Welt die Metrik nicht mehr an das Genus, sondern an die Persönlichkeiten oder an die Kunstprosa, man möchte fast sagen an die Bildung, gebunden ist. ${ }^{14}$

Demnach betrachtet schon de Groot den Klauselrhythmus bei Caesar im Vergleich zu anderen römischen Schriftstellern. Auch Shewring wendet sich im Zuge seiner mehrere antike Autoren betrachtenden klauselrhythmischen Untersuchungen einer Analyse der Schriften Caesars zu. Er kommt zu folgendem Ergebnis:

Caesar [Hervorhebung weggelassen; d. Verf.]. [...] Seeks - v - - $[\mathrm{x}]$ (16.5 per cent., slightly above Cicero's percentage), $-\mathrm{v}--\mathrm{v}[\mathrm{x}],-$ $--\mathrm{v}[\mathrm{x}],-\mathrm{v}-[\mathrm{x}],-\mathrm{v}--\mathrm{v}-[\mathrm{x}], \mathrm{v} \mathrm{v} \mathrm{v}--[\mathrm{x}],-\mathrm{v} \mathrm{v} \mathrm{v}$ $-[\mathrm{x}](3.0$ per cent.), $\mathrm{v} \mathrm{v} \mathrm{v}-\mathrm{v} \mathrm{v}[\mathrm{x}],[\mathrm{a}] \mathrm{voids}-\mathrm{v} \mathrm{v} \mathrm{v}-[\mathrm{x}], \mathrm{v} \mathrm{v} \mathrm{v}[\mathrm{x}]$, $-\mathrm{v} v-\mathrm{v}[\mathrm{x}],[\mathrm{s}]$ carcely avoids $--[\mathrm{x}]\left(20.8\right.$ per cent.). ${ }^{15}$

Sowohl de Groot als auch Shewring wenden also, nach Oberhelman, für die Untersuchung klauselrhythmischer Besonderheiten in Caesars schriftstellerischem Stil externvergleichende Studien an. ${ }^{16}$ Oberhelman bringt die Forschungsergebnisse zum Prosarhythmus bei Caesar folgendermaßen auf den Punkt:

[...] Caesar marked a unique path by synthesizing certain aspects of Cicero's style with the rhythms natural to the Latin language. ${ }^{17}$

\footnotetext{
${ }^{14}$ DE GROOT, A. W.: Der antike Prosarhythmus. Zugleich Fortsetzung des Handbook of Antique Prose-Rhythm. Groningen - Den Haag 1921, 100-101.

${ }^{15}$ SHEwring, W. H.: Prose-Rhythm and the Comparative Method. II. CQ 25.1 (1931) 15 (17.11.2011, http://www.jstor.org/stable/637096). — steht für ein longum, v für ein breve, $\mathrm{x}$ für eine syllaba anceps.

${ }^{16}$ Oberhelman unterscheidet in der Erforschung des antiken Prosarhythmus ,non-comparative' und ,comparative studies', sowie bei zweiteren die ,external' und, internal method'. Erstere beinhalten, wie es schon ihre Bezeichnung zum Ausdruck bringt, keinerlei vergleichende Analysen; sie stützen sich weder auf eine Gegenüberstellung von Schriften mehrerer antiker Autoren (external comparative) noch auf einen Vergleich der gedanklichen und damit strukturell-rhythmischen Ebenen des zu untersuchenden Textes eines bestimmten antiken Schriftstellers (internal comparative) (vgl. OBERHELMAN, S. M.: Prose Rhythm in Latin Literature of the Roman Empire - First Century B. C. to Fourth Century A. D. [Studies in Classics, Bd. XXVII]. Lewiston-Queenston-Lampeter 2003).

${ }^{17}$ Oberhelman (Anm. 16) 77.
} 
Die folgenden Untersuchungen knüpfen an die vorhandenen Erkenntnisse zum Prosarhythmus bei Caesar an. ${ }^{18}$ Das Corpus Caesarianum insgesamt wurde in der bisherigen altphilologischen Forschung noch nicht klauselrhythmisch untersucht. ${ }^{19}$ Hierzu sollen die folgenden Überlegungen und Analysen beitragen.

\section{FORSCHUNGSGEGENSTAND}

Hier werden die direkten Reden im Corpus Caesarianum nach klauselrhythmischen Gesichtspunkten analysiert und verglichen. Damit folgen die Untersuchungen in einem wichtigen Grundgedanken u. a. der Forschung de Groots und Shewrings, ${ }^{20}$ liegt ihnen doch ebenfalls eine extern-vergleichende Studie zugrunde. So geht es auch hier um einen Vergleich des Klauselrhythmus in den Werken verschiedener antiker Schriftsteller, wobei die untersuchten Textausschnitte aus dem Werk des einen Autors basierend auf bisherigen Forschungsergebnissen als klauselrhythmisch stilisiert angenommen werden können (gemeint sind Bellum Gallicum und Bellum civile), diejenigen aus der Schrift des anderen Verfassers bzw. der anderen Verfasser jedoch (noch) nicht (gemeint sind die pseudo-caesarischen Schriften). Zwar betrachten die Untersuchungen nicht die gesamten Werke Caesars und seiner Fortsetzer, jedoch sehr wohl alle direkten Reden in ebendiesen, sodass eindeutige Ergebnisse erwartet werden können.

Zahlreiche Altphilologen haben sich bereits mit der Frage nach dem Verfasser bzw. den Verfassern des Corpus Caesarianum auseinandergesetzt, so auch Barwick, Landgraf, Pötter und Seel. ${ }^{21}$ Inzwischen ist man sich in der Forschung weitgehend darüber einig, dass die pseudo-caesarischen Schriften, v. a. auch aus stilistischen Gründen, Caesar nicht zugeordnet werden sollten. Vielmehr werden Hirtius als der Verfasser des 8. Buches des Bellum Gallicum ${ }^{22}$ sowie des Bellum Alexandrinum ${ }^{23}$ und zwei uns unbekannte Autoren als Verfasser des Bellum Africum sowie des Bellum

\footnotetext{
${ }^{18}$ Für eine prägnante Zusammenfassung zu den bisherigen Forschungsergebnissen zum Prosarhythmus bei Caesar vgl. OBERHELMAN (Anm. 16) 247.

${ }^{19} \mathrm{Vgl}$. auch die neuesten Beiträge zu den pseudo-caesarischen Schriften von J. F. GAERTNER: The Corpus Caesarianum. In KREBS, C. - GRILlo, L. (Hrsg.): The Cambridge Companion to Julius Caesar, 6000 Wörter. Cambridge University Press [im Druck]; The Historical Technique, Language, and Literary Form of the Four non-Caesarian Wars. In RAAFLAUB, K. - STRASSLER, R. B. (Hrsg.): The Landmark Caesar, 2500 Wörter [im Druck]; The Origins and History of the Corpus Caesarianum. In RAAFLAUB-STRASSLER [im Druck].

${ }^{20}$ Vgl. DE GROOT, A. W.: A Handbook of Antique Prose-Rhythm. Groningen - Den Haag - Leipzig 1918; DE GRoOT (Anm. 14); Shewring, W. H.: Prose-Rhythm and the Comparative Method. $C Q$ 24.3-4 (1930) 164-173 (17.11.2011, http:// www.jstor.org/stable/637128); SHEwRING (Anm. 15).

${ }^{21}$ Vgl. vON AlBRECHT (Anm. 9) 361-363; BARWICK, K.: Caesars Commentarii und das Corpus Caesarianum [Philologus Suppl. 31.2] 1938; A. KLOTZ s. v. Iulius (Caesar). In RE IXX (1918) 272-275; LANDGRAF, G.: Untersuchungen zu Caesar und seinen Fortsetzern insbesondere über Autorschaft und Komposition des Bellum Alexandrinum und Africanum. Erlangen 1888; PÖTTER, H.: Untersuchungen zum Bellum Alexandrinum und Bellum Africanum. Stil und Verfasserfrage. Diss. Münster 1932; SEEL, O.: Hirtius. Untersuchungen über die pseudocaesarischen Bella und den Balbusbrief [Klio Beiheft 35] 1935.

${ }^{22}$ Vgl. VON ALBRECHT (Anm. 9) 361.

${ }^{23}$ Vgl. SeEL (Anm. 21) 65. Dem widerspricht von Albrecht (vgl. vON AlBRECHT [Anm. 9] 361$362)$.
} 
Hispaniense ${ }^{24}$ vermutet. Es soll geprüft werden, ob diese Annahmen mithilfe klauselrhythmischer Analysen der direkten Reden bestätigt werden können.

Auch im Hinblick auf die Verfasserfrage zum Bellum civile liegen uns Hypothesen vor. Nach Klotz können

[1]äppische Versuche, die Unechtheit des Bell[um] civ[ile] [eigene Hervorhebung; d. Verf.] zu erweisen, [...] übergangen werden. Wichtiger ist die Hypothese, da[ss] einzelne Stücke unverarbeitet aufgenommene Legatenberichte seien. Man knüpft dabei an die Darstellung der Ereignisse an, bei denen Caesar nicht zugegen gewesen ist und somit auf Berichte and[e]rer angewiesen war, und findet die Bestätigung fremden Ursprungs in gewissen sprachlichen Unterschieden zwischen diesen Partien und dem ,vollkommen caesarischen' Stil. $^{25}$

Klotz betrachtet derartige Überlegungen allerdings kritisch:

Der Beweis dafür ist nicht erbracht. Die sprachlichen Verschiedenheiten, soweit sie überhaupt Bedeutung haben, erklären sich durch den verschiedenen Zustand des Bellum Gallicum [eigene Hervorhebung; d. Verf.] und Bellum civile [eigene Hervorhebung; d. Verf.]. Sie beschränken sich ebenfalls nicht auf die fremden Verfassern zugeschriebenen Stücke. $\mathrm{Da}[\mathrm{ss}]$ bei dem skizzenhaften Zustand [...] des Bellum civile [eigene Hervorhebung; d. Verf.] der Stil der Unterlagen hie[r] und da durchschimmere, soll nicht als unmöglich bezeichnet werden. Im [A]llgemeinen wird die Annahme der unbesehenen Übernahme fremder Berichte nicht gebilligt werden können [...]. Speziell den Bericht des Curio erweist die von Caesar meisterhaft geschriebene Rede, den über die Belagerung von Massilia die eingehende Beschreibung der dem Feldherrn wohl bekannten Belagerungsmaschinen, die als Dienstberichte eines Pionieroffiziers undenkbar sind, als schriftstellerische Leistungen Caesars [... ${ }^{26}$

Deshalb werden derartige Vermutungen zur Entstehung des Bellum civile für die eigenen klauselrhythmischen Analysen nicht berücksichtigt.

\section{METHODIK}

Die eigenen klauselrhythmischen Analysen folgen den Forschungsarbeiten mehrerer Altphilologen zu Klauselrhythmus und Kolometrie. Teilweise sind sie an bisherige klauselrhythmische Untersuchungen zu einzelnen direkten Reden in den Schriften Caesars angelehnt. ${ }^{27}$

${ }^{24}$ Vgl. VON AlBrecht (Anm. 9) 361-363; SEEL (Anm. 21) 65 und 83-100.

${ }^{25}$ KLOTZ (Anm. 21) 270.

${ }^{26}$ KLOTZ (Anm. 21) 271.

${ }^{27}$ Vgl. BROADHEAD, H. D.: Latin Prose Rhythm. A New Method of Investigation. Cambridge 1922; CECI (Anm. 11); DE GroOT: A Handbook (Anm. 20); DE GROOT: Der antike Prosarhythmus (Anm. 14); 
In Anlehnung an Shewring wird das Vorkommen der folgenden quantitierenden Klauseln in den direkten Reden des Corpus Caesarianum untersucht ${ }^{28}$ :

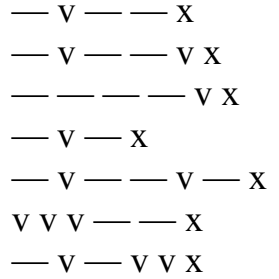

$$
\begin{aligned}
& \mathrm{V} V \mathrm{~V}-\mathrm{V} \mathrm{VX} \\
& -\mathrm{v} v \mathrm{v}-\mathrm{x} \\
& \text { V V V V v - X } \\
& -\mathrm{v} v \mathrm{v}-\mathrm{v} \mathrm{x} \\
& \mathrm{V} v \mathrm{v}-\mathrm{V} \mathrm{X} \\
& -\mathrm{vV}-\mathrm{vX} \\
& -\mathrm{v}-\mathrm{vX}
\end{aligned}
$$

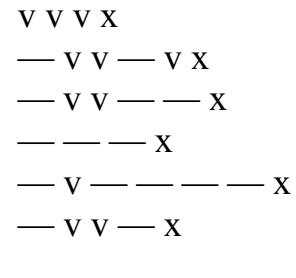

V V V X

$-\mathrm{v} v-\mathrm{x}$

Dabei wird jede ermittelte Klausel einer Stufe zugeordnet. Die Technik des Stufenvergleichs basiert auf dem

[...] [Grundg]edanke[n], Klauseln verschiedener Schlu[ss]stärke zu unterscheiden und aus dem Stufenvergleich ihre Qualitäten abzulesen, [...]. ${ }^{29}$

Zugrunde liegt die Einteilung antiker Texte in Perioden, Sätze und Kola; Perioden bestehen aus einem oder mehreren Sätzen, Sätze bestehen aus mehreren Kola. ${ }^{30}$ Eine solche Einteilung kann unterschiedlich viele Stufen beinhalten. Der Dreistufenvergleich ist in der klauselrhythmischen Forschung allerdings der gebräuchlichste ${ }^{31}$ und wird deshalb auch hier angewandt. In diesem Sinne bilden Kolongrenzen die Klauseln erster Stufe, Satzgrenzen die Klauseln zweiter Stufe und Periodengrenzen die Klauseln dritter Stufe, wobei hier das Hauptaugenmerk auf den aussagekräftigeren Klauseln der 2. und 3. Stufe liegen soll.

Für die kolometrische Analyse eines antiken Textes kann dessen Gliederung mittels Satzzeichen hilfreich sein. Da antike Texte ursprünglich jedoch keine Interpunk-

FRAENKEL, E.: Kleine Beiträge zur klassischen Philologie. Bd. I: Zur Sprache. Zur griechischen Literatur. Rom 1964; FrAENKEL, E.: Noch einmal Kolon und Satz [SBAW 1965, Heft 2]; FRAENKEL, E.: Leseproben aus Reden Ciceros und Catos [Sussidi Eruditi 22]. Rom 1968; HABINEK, TH. N.: The Colometry of Latin Prose [University of California Publications. Classical Studies, Bd. XXV]. Berkeley - Los Angeles - London 1985; Holtz (Anm. 13); Primmer, A.: Cicero numerosus. Studien zum antiken Prosarhythmus [SAWW 257, 1968]; SHEWRING (Anm. 20 und Anm. 15); STRÄTERHOFF, B.: Kolometrie und Prosarhythmus bei Cicero und Livius. De imperio Cn. Pompei und Livius 1,1-26,8 kolometrisch ediert, kommentiert und statistisch analysiert. Bd. I und II. Oelde 1995; B. STRÄTERHOFF: Kolometrie und Prosarhythmus bei Sallust am Beispiel der Rede des Caius Aurelius Cotta. In CzAPLA, B. - LEHMANN, T. LIELL, S. (Hrsg.): Vir bonus dicendi peritus. Festschrift für Alfons Weische zum 65. Geburtstag. Wiesbaden 1997, 399-406; ZANDER, K.: Eurythmia vel compositio rythmica prosae antiquae. Bd. III: Eurythmia Ciceronis. Leipzig 1914; ZIELINSKI, TH.: Das Clauselgesetz in Ciceros Reden. Grundzüge einer oratorischen Rhythmik. Philologus 9 (1904); ZIELINSKI, TH.: Der constructive Rhythmus in Ciceros Reden. Der oratorischen Rhythmik zweiter Teil. Philologus 13 (1920).

${ }^{28}$ Vgl. SHEWRING: Prose-Rhythm II (Anm. 15) 13.

${ }^{29}$ PRIMMER (Anm. 27) 107.

${ }^{30}$ Für eine ausführliche Darstellung zu Kolon und Periode vgl. LAUSBERG (Anm. 4) 458-469 ( $\S \S$ 923-947). Zum Satz als Unterteilungskategorie antiker Texte vgl. ZIELINSKI: Der constructive Rhythmus (Anm. 27) 23.

${ }^{31}$ Vgl. PrimMER (Anm. 27) und ZIELINSKI: Der constructive Rhythmus (Anm. 27). 
tion aufwiesen ${ }^{32}$ und diese somit von den Herausgebern nachträglich eingefügt worden ist, muss sie, v. a. auch im Hinblick auf klauselrhythmische Analysen, kritisch betrachtet werden. Aus diesem Grund werden die betreffenden lateinischen Texte der direkten Reden bei Caesar und den Fortsetzern seiner Schriften zunächst in diversen Textausgaben verschiedener Herausgeber sowie Erscheinungsländer und -jahre verglichen. ${ }^{33}$

${ }^{32}$ Vgl. Chatelain, É.: Paléographie des Classiques Latins. Collection de Fac-Similés. Paris 1884-1887, Tab. 46-50a. Dass dies nicht ausschließlich zutrifft, ergeben Forschungsarbeiten von Habinek, Müller und Wingo (vgl. HABINEK [Anm. 27]; MÜLLER, R. W.: Rhetorische und syntaktische Interpunktion. Untersuchungen zur Pausenbezeichnung im antiken Latein. Diss. Tübingen 1964; WINGO, E. O.: Latin Punctuation in the Classical Age [Janua linguarum, Bd. CXXXIII]. Den Haag 1972).

${ }^{33}$ Die betreffenden lateinischen Texte werden verglichen aus: I. Wissenschaftliche Textausgaben: C. Iulius Caesar, Commentarii. Bd. I: De bello Gallico. Hrsg. von R. DU PONTET [Scriptorum classicorum bibliotheca Oxoniensis]. Oxford 1988, 1. Auflage von 1900 - C. Iulius Caesar, Commentarii. Bd. II: De bello civili. De bello Alexandrino, Africo, Hispaniensi. Hrsg. von R. DU PONTET [Scriptorum classicorum bibliotheca Oxoniensis]. Oxford $1992^{17}$, Nachdruck der 1. Auflage von 1901 - C. Iulius Caesar, Commentarii. Bd. I: Commentarii belli Gallici. Hrsg. von A. KLOTZ [Bibliotheca scriptorum Graecorum et Romanorum Teubneriana]. Leipzig 1921 - C. Iulius Caesar, Commentarii rerum gestarum. Bd. I: Bellum Gallicum. Hrsg. von O. SEEL [Bibliotheca scriptorum Graecorum et Romanorum Teubneriana]. Leipzig 1961 - C. Iulius Caesar, Commentarii rerum gestarum. Bd. I: Bellum Gallicum. Hrsg. von W. HeRING [Bibliotheca scriptorum Graecorum et Romanorum Teubneriana]. Berlin 2008, Nachdruck der 1. Auflage von 1987 - C. Iulius Caesar, Commentarii. Bd. II: Commentarii belli civilis. Hrsg. von A. KLOTZ [Bibliotheca scriptorum Graecorum et Romanorum Teubneriana]. Leipzig 1969, korrigierter Nachdruck C. Iulius Caesar, Commentarii. Bd. III, Teil 1: Commentarius de bello Alexandrino. Commentarius de bello Africo. Hrsg. von B. KÜBLER [Bibliotheca scriptorum Graecorum et Romanorum Teubneriana]. Leipzig 1893 - C. Iulius Caesar, Commentarii. Bd. III, Teil 2: Commentarius de bello Hispaniensi. Hrsg. von B. KÜBLER [Bibliotheca scriptorum Graecorum et Romanorum Teubneriana]. Leipzig 1897 - C. Iulius Caesar, Commentarii. Bd. III: Commentarii belli Alexandrini, belli Africi, belli Hispaniensis. Hrsg. von A. KLOTZ [Bibliotheca scriptorum Graecorum et Romanorum Teubneriana]. Stuttgart - Leipzig 1993, unveränderter Nachdruck der 1. Auflage von 1927.

II. Textausgaben mit Kommentar: C. Iulius Caesar, Bellum Alexandrinum. Hrsg. und erklärt von R. SCHNEIDER. Berlin 1888 - C. Iulius Caesar, Bellum Africanum. Hrsg. und erklärt von R. SCHNEIDER. Berlin 1905 - C. Iulius Caesar, Commentarii de bello Gallico. Hrsg. und erklärt von F. KRANER und W. DitTENBERGER. Bde. I-III. Berlin 1913-1920 17 , vollständig überarbeitete Auflage von H. MEUSEL C. Iulius Caesar, Commentarii de bello civili. Hrsg. und erklärt von F. KRANER und F. HOFMANN. Berlin $1959^{12}$, 12. Auflage von H. MeuSEL - Iulius Caesar, The Civil War. Books I \& II. Ed. with Introduction, Translation and Commentary by J. M. CARTER. Warminster 1991 - Iulius Caesar, The Civil War. Book III. Ed. with Introduction, Translation and Commentary by J. M. CARTER. Warminster 1991.

III. Zweisprachige Textausgaben: César, Guerre civile. Éd. par P. FABRE. Vol. I: Livres I-II [Collection Guillaume Budé] Paris 1997, 1. Auflage von 1936 - César, Guerre civile. Éd. par P. FABRE. Vol. II: Livre III [Collection Guillaume Budé]. Paris 1997, 1. Auflage von 1936 - César, Guerre d' Afrique. Éd. par A. Bouvet [Collection Guillaume Budé]. Paris 1949 - César, Guerre d' Alexandrie. Éd. par J. ANDRIEU [Collection Guillaume Budé]. Paris 1983, 1. Auflage von 1954 -Caesar, Alexandrian, African and Spanish Wars. With a Translation by A. G. WAY, ed. by G. P. GoOLD [The Loeb Classical Library CDII]. Cambridge-London 1988, Nachdruck der 1. Auflage von 1955 - César, Guerre des Gaules. Éd. par L.-A. CONSTANS. Vol. I: Livres I-IV [Collection Guillaume Budé]. Paris $1958^{6}$ - César, Guerre des Gaules. Éd. par L.-A. CONSTANS. Vol. II: Livres V-VIII [Collection Guillaume Budé]. Paris $1959^{6}-$ C. Iulius Caesar, Der Bürgerkrieg. Lateinisch-Deutsch. Hrsg. von G. DORMINGER [Tusculum-Bücherei. Hrsg. von H. Färber und M. Faltner, Archiv 248]. München $1970^{3}-$ C. Iulius Caesar, Der Gallische Krieg. Lateinisch-Deutsch. Übersetzt und hrsg. von M. DEISSMANN. Stuttgart 2004.

IV. Schulausgaben: C. Iulius Caesar, Commentarii de bello Gallico. Auswahl, hrsg. von F. ECKSTEIN [Altsprachliche Textausgaben, Heft 5]. Frankfurt a. M. 1988 ${ }^{14}$; Caesar, Weltherrscher. Ein literarisches Porträt. Bearbeitet von F. MAIER, Zeichnungen von A. BELL [Antike und Gegenwart. Lateinische Texte zur Erschließung europäischer Kultur, hrsg. von F. MAIER]. Bamberg 2007. 
Schließlich werden diejenigen Textstellen, bei denen Unterschiede in der Interpunktion auffallen, im Hinblick auf diese diskutiert, kann man doch davon ausgehen, dass diesbezügliche Differenzen auf unterschiedliche Interpretationsansätze zurückzuführen sind, die so wiederum Auswirkungen auf den Klauselrhythmus haben können.

Die folgende Übersicht fasst die wesentlichen Arbeitsschritte bei den durchgeführten klauselrhythmischen Analysen zusammen:

1) Vergleich der betreffenden lateinischen Texte der direkten Reden bei Caesar und den Fortsetzern seiner Schriften aus diversen Textausgaben verschiedener Herausgeber sowie Erscheinungsländer und -jahre.

2) Diskussion derjenigen Textstellen, bei denen Unterschiede in der Interpunktion auffallen, anschließend Festlegung der Interpunktion anhand eigener Satzanalyse und Interpretation.

3) Festlegung von Kolon-, Satz- und Periodenschlüssen mittels Kolometrie und Interpretation. (Bei unechten [Partizipialkonstruktionen, Gerundialia, AcI, NcI] und echten Gliedsätzen sowie bei Appositionen werden zunächst immer Kolongrenzen angenommen, ebenso bei Bestandteilen einer Aufzählung oder einer Gegenüberstellung mehrgliedriger Wortgruppen. ${ }^{34}$ )

4) Bestimmung langer und kurzer Silben gemäß der natürlichen Quantitätenverteilung innerhalb der Worte ${ }^{35}$ sowie nach den Regeln der lateinischen Metrik und Prosodie. $^{36}$

5) Festlegung der Stufeneinteilung.

6) Zählung der einzelnen Klauseln in der jeweiligen Stufe (hier: Angabe der Anzahl der einzelnen Klauseln). ${ }^{37}$

7) Auswertung der Ergebnisse.

${ }^{34}$ Vgl. MENGE, H.: Lehrbuch der lateinischen Syntax und Semantik. Völlig neu bearbeitet von TH. BURKARD - M. SCHAUER. Darmstadt $2007^{3}$, unveränderter Nachdruck der 2., überarbeiteten Auflage von 2005. Vgl. hier auch die Definition der oratio recta im Unterschied zur oratio obliqua.

${ }^{35}$ Mithilfe von: Ausführliches Lateinisch-Deutsches Handwörterbuch. Ausgearbeitet von K. E. GEORGES, Bd. I und II. Hannover $1995^{8}$, unveränderter Nachdruck der 8. verbesserten und vermehrten Auflage von H. GeORGES; Oxford Latin Dictionary. Ed. by P. G. W. Glare. Oxford 1992, Neudruck der 1. Auflage von 1982; RubenBAUER H. - HofmAnN J. B.: Lateinische Grammatik. Neubearbeitet von R. HEINE. Bamberg - München $1995^{12}$; Thesaurus linguae Latinae. Leipzig 1905.

${ }^{36}$ Vgl. BOLDRINI, S.: Prosodie und Metrik der Römer. Aus dem Italienischen übertragen von B. W. HÄUPTLI. Stuttgart-Leipzig 1999; CrUSIUS, F.: Römische Metrik. Eine Einführung. Neu bearbeitet von H. RubenBAUER. München $1992^{8}$, 4. Nachdruck der 8. Auflage; HALPORN, J. W. - OSTWALD, M.: Lateinische Metrik. Göttingen $2004^{5}$, unveränderte Auflage.

${ }^{37}$ An dieser Stelle sei darauf hingewiesen, dass es in dem Hirtius zugeschriebenen 8. Buch des Bellum Gallicum keine direkten Reden gibt. Deshalb kann das 8. Buch des Bellum Gallicum in diese Forschungsarbeit nicht einbezogen werden.

Für die Ermittlung und Zählung der Klauseln stammen die betreffenden lateinischen Texte aus: C. Iulius Caesar, Commentarii rerum gestarum. Bd. I: Bellum Gallicum. Hrsg. von W. HeRING [Bibliotheca scriptorum Graecorum et Romanorum Teubneriana]. Berlin 2008, Nachdruck der 1. Auflage von 1987 - C. Iulius Caesar, Commentarii. Bd. II: Commentarii belli civilis. Hrsg. von A. KLOTZ [Bibliotheca scriptorum Graecorum et Romanorum Teubneriana]. Leipzig 1969, korrigierter Nachdruck - C. Iulius Caesar, Commentarii. Bd. III: Commentarii belli Alexandrini, belli Africi, belli Hispaniensis. Hrsg. von A. KLOTZ [Bibliotheca scriptorum Graecorum et Romanorum Teubneriana]. Stuttgart-Leipzig 1993, unveränderter Nachdruck der 1. Auflage von 1927. 


\section{TEXTBEISPIELE}

Beispiel I: Critognatus (Caes. Gall. 7. 77. 3ff.)

nihil inquit de eorum sententia dicturus sum $(--\ldots \mathrm{x})$ qui turpissimam servitutem deditionis nomine appellant $(-\mathrm{v}--\mathrm{x})$ neque hos habendos civium loco $(\mathrm{v}-\mathrm{v}$ $\mathrm{x})$ neque ad concilium adhibendos censeo $(--\mathrm{v} x)$ cum his mihi res sit qui eruptionem probant $(--\mathrm{v} x)$ quorum in consilio omnium vestrum consensu pristinae residere virtutis memoria videtur $(\mathrm{v} v \mathrm{v} v \mathrm{v}-\mathrm{x})$ animi est ista mollitia non virtus $(\mathrm{v} v \mathrm{v}-\mathrm{x})$ paulisper inopiam ferre non posse $(-\mathrm{v}-\mathrm{x}$ ) qui se ultro morti offerant facilius reperiuntur $(-\mathrm{v} v \mathrm{v}-\mathrm{x})$ quam qui dolorem patienter ferant $(--\mathrm{vx}) \ldots$

Beispiel II: Curio (Caes. civ. 2. 31. 2ff.)

qua enim inquit fiducia et opere et natura loci munitissima castra expugnari posse confidimus (- $-\mathrm{v} x)$ aut vero quid proficimus si accepto magno detrimento ($-\mathrm{x})$ ab oppugnatione castrorum discedimus ( $-\mathrm{v}$ x) quasi non et felicitas rerum gestarum exercitus benevolentiam imperatoribus $(--\mathrm{v} x)$ et res adversae odia concilient $(-\mathrm{v} v \mathrm{x})$ castrorum autem mutatio quid habet nisi turpem fugam et desperationem omnium (- $-\mathrm{vx})$ et alienationem exercitus $(--\mathrm{vx}) \ldots$

Beispiel III: Euphranor (Bell. Alex. 15. 3f.)

videris mihi inquit Caesar vereri $(-\mathrm{v}-\mathrm{x})$ si haec vada primis navibus intraris ($\mathrm{v} v--\mathrm{x})$ ne prius dimicare cogaris $(-\mathrm{v}--\mathrm{x})$ quam reliquam classem potueris explicare $(-\mathrm{v}-\mathrm{x})$ nobis rem committe nos proelium sustinebimus $(\mathrm{v}-\mathrm{v} \mathrm{x})$ neque tuum iudicium fallemus dum reliqui subsequantur $(-v-x)$ hos quidem diutius in nostro conspectu gloriari $(-\mathrm{v}-\mathrm{x})$ magno nobis et dedecori et dolori est $(-\mathrm{v}-\mathrm{x})$

Beispiel IV: Caesar (Bell. Afr. 54. 2ff.)

... Tite Saliene M. Tiro C. Clusinas cum ordines in meo exercitu beneficio ( $\mathrm{v} v \mathrm{v} \mathrm{x}$ ) non virtute [sitis] consecuti ita vos gesseritis $(-\mathrm{v} v \mathrm{x})$ ut neque bello fortes neque pace boni aut utiles fueritis ( $\mathrm{v} v \mathrm{v}$ x) et magis in seditione concitandisque militibus adversum vestrum imperatorem $(-\mathrm{v}-\mathrm{x}$ ) quam pudoris modestiaeque $(-\mathrm{v}-$ $\mathrm{x})$ fueritis studiosiores $(-\mathrm{v}-\mathrm{x})$ indignos vos esse arbitror $(---\mathrm{x})$ qui in meo exercitu ordines ducatis missosque facio $(\mathrm{v} \vee \mathrm{v} x)$ et quantum pote abesse ex Africa iubeo $(-\mathrm{v} v \mathrm{x})$

Beispiel V: Tibullius (Bell. Hisp. 17. 1f.)

utinam quidem di inmortales fecissent (- $-\mathrm{x}$ ) ut tuus potius miles quam $\mathrm{Cn}$. Pompei factus essem $(-\mathrm{v}-\mathrm{x})$ et hanc virtutis constantiam in tua victoria $(--\mathrm{v}$ 
$\mathrm{x})$ non in illius calamitate praestarem $(-\mathrm{v}--\mathrm{x})$ cuius funestae laudes quoniam ad hanc fortunam reciderunt $(-\mathrm{v} \mathrm{v}-\mathrm{x})$ ut cives Romani indigentes praesidii et propter patriae luctuosam perniciem ducimur hostium numero ( $-\mathrm{v} v \mathrm{x}$ ) qui neque in illius prospera acie primam fortunam $(--\ldots \mathrm{x})$ neque in adversa secundam obtinuimus ( $\mathrm{v}$ v v x) ...

\section{ERGEBNISSE UND AUSWERTUNG}

\begin{tabular}{|c|c|c|c|}
\hline Klauselende & $B G$ & $B C$ & $P s C$ \\
\hline$\ldots v--x$ & 5 & 2 & 1 \\
\hline$\ldots--\mathrm{vx}$ & 2 & 2 & ----- \\
\hline$\ldots-\mathrm{v}-\mathrm{x}$ & 3 & 1 & ---- \\
\hline$\ldots-\mathrm{v} v \mathrm{x}$ & 2 & 3 & 1 \\
\hline$\ldots \mathrm{v} \mathrm{v}-\mathrm{x}$ & 3 & ---- & ---- \\
\hline$\ldots \mathrm{v}-\mathrm{v} x$ & 1 & ---- & ---- \\
\hline$\ldots \mathrm{v} v \mathrm{vx}$ & ----- & ----- & ----- \\
\hline$\ldots---x$ & 2 & 1 & ----- \\
\hline
\end{tabular}

Tab. 1: Klauseln der 2. Stufe (BG-Bellum Gallicum, BC-Bellum civile, $P s C$ - die pseudo-caesarischen Schriften)

\begin{tabular}{|c|c|c|c|}
\hline Klauselende & $B G$ & $B C$ & $P s C$ \\
\hline$\ldots v--x$ & 6 & 6 & 8 \\
\hline$\ldots--\mathrm{vx}$ & 10 & 11 & ----- \\
\hline$\ldots-\mathrm{v}-\mathrm{x}$ & 12 & 12 & 8 \\
\hline$\ldots-\mathrm{v} \mathrm{v} \mathrm{x}$ & 4 & 9 & 3 \\
\hline$\ldots \mathrm{V} \mathrm{v}-\mathrm{x}$ & 1 & 8 & 5 \\
\hline$\ldots \mathrm{v}-\mathrm{v} \mathrm{x}$ & ----- & 6 & 1 \\
\hline$\ldots \mathrm{V} v \mathrm{v} x$ & 1 & 2 & 1 \\
\hline$\ldots---\mathrm{x}$ & 3 & 9 & 7 \\
\hline
\end{tabular}

Tab. 2: Klauseln der 3. Stufe (BG-Bellum Gallicum, BC-Bellum civile, $P s C$ - die pseudo-caesarischen Schriften)

Die vorliegenden Ergebnisse zeigen zum einen deutliche Unterschiede in der Stufung und damit in der gedanklich-stilistischen Struktur der direkten Reden in den Kommentarien Caesars im Vergleich zu den direkten Reden in den pseudo-caesarischen 
Schriften auf. Denn in den pseudo-caesarischen Schriften treten bei annähernd gleicher Textmenge insgesamt deutlich weniger Satzschlüsse und damit Klauseln der 2. Stufe auf als in den Kommentarien Caesars. Während Caesar in den direkten Reden also übersichtlich gestaltete Perioden bevorzugt, die sich des öfteren der besseren Verständlichkeit wegen aus mehreren kurzen Sätzen zusammensetzen, wählen seine Fortsetzer hier zumeist lange, unübersichtliche Perioden, die sich insgesamt nur zweimal in mehrere Sätze untergliedern.

Zum anderen sind signifikante Unterschiede in der Verwendung einzelner Klauseln auf der 2. und 3. Stufe zu verzeichnen. So kommen alle Klauseln, die auf ... — v x enden (nach Shewring die Klauseln — v - - v x , - - - - v x, v v v - v x, - v v - — v x, ) in den direkten Reden des Bellum Gallicum und Bellum civile auf der 2. und 3. Stufe häufig vor, während sie in denen der pseudo-caesarischen Schriften auf der 2. und 3. Stufe überhaupt keine Verwendung finden. ${ }^{38}$ Während Caesar in den direkten Reden also, neben wenigen anderen Klauseln, für Satz- und Periodenschlüsse Klauseln, die auf ... —— v x enden, deutlich bevorzugt, meiden seine Fortsetzer diese hier konsequent.

\title{
SCHLUSSFOLGERUNG
}

Die Gegenüberstellung der Klauseln der 2. und 3. Stufe in den direkten Reden des Bellum Gallicum und Bellum civile auf der einen und denen der pseudo-caesarischen Schriften auf der anderen Seite ergibt signifikante Unterschiede, aus denen sich ein weiteres Argument hinsichtlich der Verfasserfrage gewinnen lässt. Die vorliegenden Ergebnisse beweisen, dass anhand der aufgezeigten klauselrhythmischen Analysen der direkten Reden in den Werken Caesars und denen seiner Fortsetzer valide Aussagen bezüglich der Verfasserfrage des Corpus Caesarianum getroffen werden können. Es lassen sich auf Satz- und Periodenebene eindeutige klauselrhythmische Unterschiede zwischen den direkten Reden in den Kommentarien Caesars und denen in den pseudo-caesarischen Schriften festmachen. So werden die bisherigen Annahmen zur Autorschaft der Schriften Bellum Alexandrinum, Bellum Africum und Bellum Hispaniense durch die vorliegenden Ergebnisse bestätigt, insofern dass diese drei Werke Caesar nicht zuzuordnen sind.

\author{
Kathleen Börner, M. A. \\ Friedrich-Schiller-Universität Jena \\ Fürstengraben 1 \\ 07743 Jena \\ Deutschland \\ kathleen.boerner@gmx.net
}

\footnotetext{
${ }^{38}$ Bei den Klauseln der 2. Stufe sind zwar noch weitere Unterschiede zwischen den direkten Reden der Kommentarien Caesars und denen der pseudo-caesarischen Schriften zu erkennen, diese sind aber von geringem Ausmaß und deshalb als nicht signifikant zu beurteilen. Aus diesem Grund werden sie bei der Auswertung nicht berücksichtigt.
} 